\title{
JACOBIAN ELLIPTIC FUNCTIONS AND MINIMAL SURFACES
}

\author{
FARUK F. ABI-KHUZAM
}

(Communicated by Peter Li)

\begin{abstract}
Using Jacobian elliptic functions we construct families of complete immersed minimal surfaces, with one, two or three ends and a non-trivial symmetry group.
\end{abstract}

Let $M$ be a minimal surface of genus $g$ and finite total curvature $C(M)$. A considerable amount of work has gone into the problem of constructing examples of such a surface with a given $C(M)$ and prescribed ends [1], [2], [3], [7]. As pointed out in [6] such examples would be of interest in connection with various problems about minimal surfaces, e.g., the question of distribution of values of the Gauss-map of the surface. If $M$ is complete, oriented and of genus one, then it is classical [5], [6] that $M$ is determined by a triple $\{R, f(z) d z, g(z)\}$ where $R$, the space for isothermal parameters, equals a torus $T^{2}=\mathbb{C} / L$, formed with respect to a lattice $L$, from which a finite number of points have been removed, i.e., $R=T^{2}-\left\{p_{1}, p_{2}, \ldots, p_{n}\right\}$; and $f(z) d z$ is a holomorphic differential on $R$ and $g$ is a meromorphic function on $R$. Through the canonical projection $\pi: \mathbb{C} \rightarrow \mathbb{C} / L, f$ and $g$ can be identified with elliptic functions on $\mathbb{C}$ having the same period lattice $L$. If $\mathscr{P}$ is the Weierstrass-elliptic function associated to the lattice $L$, then any elliptic function with period lattice $L$ may be expressed in terms of $\mathscr{P}$ and $\mathscr{P} \prime$. This is, perhaps, the reason why most known examples of genus one minimal surfaces [1], [2], [3], [7] employ explicitly the Weierstrass $\mathscr{P}$-function. Costa's surface [2] is one such example where $f(z)=\mathscr{P}(z)$ and $g(z)=\alpha / \mathscr{P}^{\prime}(z)$ for some constant $\alpha$.

In this paper we use Jacobian elliptic functions to construct three families of minimal surfaces of genus one:

1. complete minimal surfaces, conformally the square torus with one point removed and total curvature $-4 \pi(2 n+1), n=2,3, \ldots$;

2. complete minimal surfaces, conformally the square torus with two points removed and total curvature $-4 \pi(2 n+1)$;

3. complete minimal surfaces, conformally the square torus with three points removed and total curvature $-4 \pi(2 n+3)$.

Received by the editors April 28, 1994.

1991 Mathematics Subject Classification. Primary 53A10. 
All surfaces enjoy certain geometric properties among which we note:

- The Gauss map composed with sterographic projection is a constant multiplied by a simple combination of three Jacobian functions.

- To each surface there corresponds a group of motions of $R^{3}$ under which it is invariant. The group is generated by

$$
\left[\begin{array}{ccc}
0 & -1 & 0 \\
1 & 0 & 0 \\
0 & 0 & (-1)^{n+1}
\end{array}\right]
$$

and

$$
\left[\begin{array}{ccc}
1 & 0 & 0 \\
0 & -1 & 0 \\
0 & 0 & \varepsilon
\end{array}\right] \quad(\varepsilon= \pm 1)
$$

\section{THE WEIERSTRASS REPRESENTATION}

Corresponding to a parameter $k, 0<k<1$, let $K$ and $E$ be the complete elliptic integrals of the first and second kinds. That is,

$$
K=\int_{0}^{\pi / 2} \frac{d \theta}{\sqrt{1-k^{2} \sin ^{2} \theta}}, \quad E=\int_{0}^{\pi / 2} \sqrt{1-k^{2} \sin ^{2} \theta} d \theta .
$$

It is the usual practice to refer to $k$ as the modulus. If $k^{\prime}$ is the complementary modulus, that is $k^{\prime}>0$ and $k^{\prime 2}+k^{2}=1$, then we define $K^{\prime}$ and $E^{\prime}$ respectively as the same functions of $k^{\prime 2}$ as $K$ and $E$ are of $k^{2}$. Using $K$ and $K^{\prime}$ we define the lattice

$$
L\left(K, K^{\prime}\right)=\left\{2 K m+2 i K^{\prime} n: m, n \in \mathbb{Z}\right\}
$$

and let

$$
\bar{R}=\mathbb{C} / L\left(K, K^{\prime}\right)
$$

be the corresponding torus. We take $M=\bar{R}-\left\{p_{1}, p_{2}, \ldots, p_{n}\right\}$ as the domain for isothermal parameters and, through the canonical projection $\pi: \mathbb{C} \rightarrow \bar{R}$, identify meromorphic functions on $\bar{R}$ with elliptic functions on $\mathbb{C}$ having $L\left(K, K^{\prime}\right)$ as their period lattice. If $f$ and $g$ are two such functions, then a mapping $X: M \rightarrow \mathbb{R}^{3}$ defined by

$$
X(z)=\operatorname{Re} \int^{z} \frac{1}{2}\left(1-g^{2}, i\left(1+g^{2}\right), 2 g\right) f d z
$$

will be a regular complete minimal immersion of genus one and $n$ ends, if $f$ and $g$ satisfy the following:

(a) $f$ is analytic on $M, g$ is meromorphic on $M$ and every zero of $f$ on $M$ coincides with a pole of $g$ so that its order as a zero of $f$ is twice its order as a pole of $g$;

(b) (Residues) $\operatorname{Res}_{p_{\nu}} f(z) g(z) \in \mathbb{R}$ and $\overline{\operatorname{Res}_{p_{\nu}} f(z)}+\operatorname{Res}_{p_{\nu}} f g^{2}(z)=0$ for $\nu=1,2,3, \ldots, n$. then

(c) (Integrals) If $\gamma_{1}$ and $\gamma_{2}$ are generators of the fundamental group of $\bar{R}$,

$$
\operatorname{Re} \int_{\gamma_{j}} g(z) f(z) d z=0 \text { and } \overline{\int_{\gamma_{j}} f(z) d z}=\int_{\gamma_{j}} f(z) g^{2}(z) d z \quad \text { for } j=1,2 \text {. }
$$


(d) (Completeness) $\int_{l}\left(1+|g(z)|^{2}|f(z)||d z|=\infty\right.$ for each divergent path $l$ in $\bar{R}$.

The representation (2.3) is called the Weierstrass representation [6] and the above conditions (a), (b), (c) and (d) may be found in [1], [5], [6].

\section{SURFACES WITH ONE OR TWO ENDS}

Theorem 1. Let $\operatorname{sn}(z, k), c n(z, k)$ and $d n(z, k)$ be the Jacobian elliptic functions to the modulus $k$. Let $k^{\prime}$ be the complementary modulus and

$$
K=\int_{0}^{\pi / 2} \frac{d \theta}{\sqrt{1-k^{2} \sin ^{2} \theta}}, \quad K^{\prime}=\int_{0}^{\pi / 2} \frac{d \theta}{\sqrt{1-k^{\prime 2} \sin ^{2} \theta}} .
$$

Let $T^{2}=\mathbb{C} / L\left(K, K^{\prime}\right)$ where

$$
L\left(K, K^{\prime}\right)=\left\{2 n K+2 i m K^{\prime}: m, n \in \mathbb{Z}\right\} .
$$

Let $p_{0}, p_{1}, p_{2}$ and $p_{3}$ be the points in $T^{2}$ corresponding to $0, K, K+i K^{\prime}$ and $i K^{\prime}$. Let

$$
f(z)=c n^{4 n+2}(z, k), \quad g(z)=\alpha s n(z, k) d n(z, k) / c n^{2 n+1}(z, k)
$$

where $n$ is an integer and $\alpha=\alpha(n)$ is a constant defined by

$$
\begin{array}{ll}
\alpha^{2}=\frac{3}{2 K} \int_{0}^{2 K} c n^{4 n+2} t d t & (n \geq 0), \\
\alpha^{2}=\frac{-3}{2 K} \int_{0}^{2 K} c n^{4 m-2} t d t & (m=-n \geq 2) .
\end{array}
$$

Assume $k=\frac{1}{\sqrt{2}}$ and let $X: D \rightarrow \mathbb{R}^{3}$ be defined by the Weierstrass representation formula (2.3) with $f$ and $g$.

(a) If $D=T^{2}-\left\{p_{3}\right\}$ and $n \geq 0$, then $X$ is a regular conformal minimal immersion and the surface $M=X(D) \subset \mathbb{R}^{3}$ is complete with total curvature $-8 \pi$ if $n=0$ and $-4 \pi(2 n+1)$ if $n=1,2,3, \ldots$.

(b) If $D=T^{2}-\left\{p_{1}, p_{3}\right\}$ and $n \leq-2$, then $X$ is a regular conformal minimal immersion and $M$ is complete with total curvature $-4 \pi(2|n|+1)$.

Note. Since $k=\frac{1}{\sqrt{2}}$, we have $k^{\prime}=k$ and $K=K^{\prime}$. In the sequel we will suppress $k$ and write e.g. $s n z$ instead of $s n(z, k)$; but use of identities will be easier to follow if we continue to write $K, K^{\prime}$ forgetting the fact that they are equal. The constants $4 K$ and $4 i K^{\prime}$ are not basic periods for $s n z, c n z$ and $d n \quad z$. But in view of the properties $[8$, p. 503]

$$
s n(z+2 K)=-s n z, \quad c n(z+2 K)=-c n z, \quad d n(z+2 K)=d n z
$$

and

(3.5) $\operatorname{sn}\left(z+2 i K^{\prime}\right)=\operatorname{sn} z, \quad c n\left(z+2 i K^{\prime}\right)=-c n z, \quad d n\left(z+2 i K^{\prime}\right)=-d n z$

one sees immediately that the functions $f$ and $g$ defined by (3.2) are, for each integer $n$, doubly periodic with periods $2 K$ and $2 i K^{\prime}$. Their zeros and poles in a fundamental region (shown below as a rectangle!) may be depicted as in Figure 1. 


$$
\begin{array}{cccccc}
i K^{\prime} \cdot \infty^{4 n+2} & \cdot & \cdot & \cdot & \cdot & \cdot \\
\cdot & 0^{4 n+2} & \cdot & \cdot 0^{1} & \cdot 0^{1} & \cdot
\end{array}
$$

$\left(n \geq 0, \infty^{-1}=0,0^{-1}=\infty\right)$.

$$
\begin{array}{cccccc}
i K^{\prime} \cdot 0^{4 m-2} & \cdot & \cdot & \cdot & \cdot & \bullet \\
\cdot & \cdot \infty^{4 m-2} & \cdot & \cdot \infty^{2 m+1} & \cdot 0^{\prime} & \cdot
\end{array}
$$

$(n=-m \leq-2)$.

FIGURE 1

Proof. (a) If $n \geq 1$, then the only pole of $g$ in the fundamental region occurs at $K$ with multiplicity $2 n+1$. But $f$ has a zero at $K$ with multiplicity $4 n+2=2(2 n+1)$. If $n=0, g$ has two simple poles at $K$ and $i K^{\prime}$. The pole at $i K^{\prime}$ corresponds to $p_{3}$ and $D=T^{2}-\left\{p_{3}\right\}$. The pole of $g$ at $K$ is now simple and $f$ has a double zero there and no other zeros in the fundamental region.

Consider next the residues of $f, f g$ and $f g^{2}$ at $i K^{\prime}$. We have [8, p. 503]

(3.6) $\operatorname{sn}\left(z+i K^{\prime}\right)=\frac{1}{k \operatorname{snz}}, \quad c n\left(z+i K^{\prime}\right)=\frac{-i d n z}{k \operatorname{snz}}, \quad d n\left(z+i K^{\prime}\right)=\frac{-i c n z}{\operatorname{snz}}$.

Since $s n z$ is an odd function and $c n z$ and $d n z$ are even functions, it follows that $f\left(z+i K^{\prime}\right), f g\left(z+i K^{\prime}\right)$ and $f g^{2}\left(z+i K^{\prime}\right)$ are all even functions of $z$ and so each must have zero residue at zero. Thus

$$
\operatorname{Res}_{i K^{\prime}} f=\operatorname{Res}_{i K^{\prime}} f g=\operatorname{Res}_{i K^{\prime}} f g^{2}=0 \text {. }
$$

Consider next the period integrals of $f, f g$ and $f g^{2}$. For this let us introduce the two paths $\gamma_{1}$ and $\gamma_{2}$ defined by

$$
z(t)=i \frac{K^{\prime}}{2}+t, \quad 0 \leq t \leq 2 K, \quad \text { and } \quad z(t)=\frac{K}{2}+i t, \quad 0 \leq t \leq 2 K^{\prime},
$$

respectively. Cauchy's theorem and periodicity make it possible to move $\gamma_{1}$ down to the real segment $[0,2 K]$ and $\gamma_{2}$ to the vertical segment from $K$ to $K+2 i K^{\prime}$ so that

$$
\int_{\gamma_{1}} f(z) d z=\int_{0}^{2 K} c n^{4 n+2} t d t \text { and } \int_{\gamma_{2}} f(z) d z=\int_{K}^{K+2 i K^{\prime}} f(\zeta) d \zeta
$$

where the path of integration in the second integral is the vertical line segment. Now the transformation

$$
c n(K+i t, k)=\frac{i k^{\prime}}{k} c n\left(K^{\prime}+t, k^{\prime}\right)
$$

gives $c n(K+i t)=i c n(K+t)$ since we are assuming $k=k^{\prime}$. Thus

$$
\begin{aligned}
\int_{\gamma_{2}} f(z) d z & =\int_{0}^{2 K^{\prime}} i f(K+i t) d t \\
& =-i \int_{0}^{2 K^{\prime}} c n^{4 n+2}\left(K^{\prime}+t\right) d t=-i \int_{0}^{2 K} c n^{4 n+2} t d t .
\end{aligned}
$$


Next we have, since the derivative of $c n z$ is $-\operatorname{sn} z d n z$,

$$
\int f(z) g(z) d z=\frac{-\alpha}{2 n+2} c n^{2 n+2} z
$$

so that

$$
\int_{\gamma_{j}} f(z) g(z) d z=0 \text { for } j=1,2
$$

by periodicity of $c n z$.

For the integral of $f g^{2}$ we have

$$
\int f(z) g^{2}(z) d z=\alpha^{2} \int s n^{2} z d n^{2} z d z=\frac{\alpha^{2}}{3}\{z-\operatorname{sn} z \operatorname{cn} z d n z\}
$$

as can be easily verified using the differentiation formulas

$$
\frac{d(\operatorname{sn} z)}{d z}=c n z d n z, \quad \frac{d(d n z)}{d z}=-k^{2} \operatorname{sn} z \operatorname{cn} z
$$

together with the identities

$$
s n^{2} z+c n^{2} z=1, \quad d n^{2} z+k^{2} s n^{2} z=1
$$

and the assumption $k=\frac{1}{\sqrt{2}}$.

It follows that

$$
\int_{\gamma_{1}} f(z) g^{2}(z) d z=\frac{2 K \alpha^{2}}{3} \text { and } \int_{\gamma_{2}} f(z) g^{2}(z) d z=\frac{2 i K^{\prime} \alpha^{2}}{3} .
$$

In view of the definition of $\alpha^{2}$ and the equality $K=K^{\prime}$ we have

$$
\overline{\int_{\gamma_{1}} f(z) d z}=\int_{0}^{2 K} c n^{4 n+2} t d t=\frac{2 K \alpha^{2}}{3}=\int_{\gamma_{1}} f(z) g^{2}(z) d z
$$

and

$$
\overline{\int_{\gamma_{2}} f(z) d z}=i \int_{0}^{2 K} c n^{4 n+2} t d t=\frac{2 i K \alpha^{2}}{3}=\int_{\gamma_{2}} f(z) g^{2}(z) d z .
$$

It remains to verify completeness, but this is immediate since $f$ has a pole at $i K^{\prime}$ of order $4 n+2$ and $g$ has a pole at $i K^{\prime}$ of order $2 n-1$ if $n \geq 1$ and a simple zero if $n=0$. This shows that $X$ defined by the Weierstrass representation (2.3) with $f$ and $g$ is a regular conformal minimal immersion and that $M=X(D)$ is complete. Finally, since $g$ is of order $2 n+1$ when $n \geq 1$ and of order 2 when $n=0$, the curvature of $M$ will be $-4 \pi(2 n+1)$ in the first case and $-8 \pi$ in the second. This finishes the proof of part (a).

(b) Write $m=-n$ so that, since $n \leq-2$,

$$
f(z)=\frac{1}{c n^{4 m-2} z} \text { and } g(z)=\alpha c n^{2 m-1} z \operatorname{sn} z d n z .
$$

Since $D=T^{2}-\left\{p_{1}, p_{2}\right\}$, both $f$ and $g$ are analytic in the corresponding region and $f$ is never zero there.

It is clear that $\operatorname{Res}_{i K^{\prime}} f=\operatorname{Res}_{i K^{\prime}} f g=0$ since both are regular at $i K^{\prime}$ and that $\operatorname{Res}_{i K^{\prime}} f g^{2}=0$ since $f g^{2}\left(z+i K^{\prime}\right)$ is an even function of $z$. Using the identities [8, p. 500]

(3.20) $\operatorname{sn}(z+K)=\frac{c n z}{d n z}, \quad c n(z+K)=-\frac{k^{\prime} s n z}{d n z} \quad$ and $\quad d n(z+K)=\frac{k^{\prime}}{d n z}$ 
one sees that $f(z+K), f g(z+K)$ and $f g^{2}(z+K)$ are all even functions of $z$ and so must have residue zero at zero. (Of course $f g^{2}$ is regular at $K$.) Thus

$$
\operatorname{Res}_{K} f=\operatorname{Res}_{K} f g=\operatorname{Res}_{K} f g^{2}=0 .
$$

Consider next the integrals of $f, f g$ and $f g^{2}$. Since $f$ has now a pole at $K$, we use Cauchy's theorem and periodicity to move the line of integration so that it goes from $i K^{\prime}$ to $2 K+i K^{\prime}$. By (3.6) and (3.20) we have $c n\left(t+i K^{\prime}\right)=$ $i / c n(t+K)$ since we are assuming $k=k^{\prime}$. Thus

$$
\begin{aligned}
\int_{\gamma_{1}} f(z) d z & =\int_{0}^{2 K} f\left(i K^{\prime}+t\right) d t \\
& =-\int_{0}^{2 K} c n^{4 m-2}(t+K) d t=-\int_{0}^{2 K} c n^{4 m-2} t d t
\end{aligned}
$$

On the other hand

$$
\begin{aligned}
\int_{\gamma_{2}} f(z) d z & =\int_{0}^{2 K^{\prime}} f(i t) i d t \\
& =i \int_{0}^{2 K^{\prime}} c n^{-4 m+2}(i t) d t=i \int_{0}^{2 K^{\prime}} c n^{4 m-2} t d t,
\end{aligned}
$$

since $(c n(i t, k))^{-1}=c n\left(t, k^{\prime}\right)=c n(t, k)$ by $[8$, p. 505] and the assumption $k=k^{\prime}$.

Next we have

$$
\int f(z) g(z) d z=\frac{-\alpha c n^{-2 m+2} z}{(2 m-2)}, \quad m \geq 2 .
$$

Thus

$$
\int_{\gamma_{j}} f(z) g(z) d z=0 \text { for } j=1,2 \text { and } m \geq 2 \text {, }
$$

by periodicity of $c n^{-2 m+2}$. The integrals $\int_{\gamma_{j}} f(z) g^{2}(z) d z$ continue to be given by (3.13) with the appropriate $\alpha$ since $f(z) g^{2}(z)=\alpha^{2} s n^{2} z d n^{2} z$. It is now clear in view of (3.22), (3.23) and (3.13) that the value

$$
\alpha^{2}=\frac{-3}{2 K} \int_{0}^{2 K} c n^{4 m-2} t d t
$$

gives

$$
\overline{\int_{\gamma_{j}} f(z) d z}=\int_{\gamma_{j}} f(z) g^{2}(z) d z \quad \text { for } j=1,2 .
$$

We conclude that $X$ defined by the Weierstrass representation with $f$ and $g$ on $D-\left\{p_{1}, p_{3}\right\}$ is a regular conformal minimal immersion. Since $f$ and $g$ both have poles at $i K^{\prime}$ while, at $K, f$ has a pole of order $4 m-2$ and $g$ a zero of order $2 m-1$, we may conclude that $M=X(D)$ is a complete surface. Finally since $g$ is of order $2 m+1$, the total curvature of $M$ is $-4 \pi(2 m+1)$.

\section{SURFACES WITH THREE ENDS}

Theorem 2. In the notation of Theorem 1, let

$$
f(z)=c n^{4 n+2} z, \quad g(z)=\beta / c n^{2 n+1} z \operatorname{sn} z d n z
$$


where $\beta=\beta(n)$ is a constant defined by

$$
\beta^{2}=\frac{1}{2 K} \int_{0}^{2 K} c n^{4 n+2} t d t \quad(n \geq 0),
$$

and assume that the modulus $k=\frac{1}{\sqrt{2}}$.

(a) If $D=T^{2}-\left\{p_{0}, p_{2}, p_{3}\right\}$ and $n \geq 0$, then $X$ is a regular conformal minimal immersion and the surface $M=X(D) \subseteq \mathbb{R}^{3}$ is complete with total curvature $-4 \pi(2 n+3)$.

(b) If we write $X=\left(X_{1}, X_{2}, X_{3}\right)$, then for $n=0$,

$$
\begin{aligned}
& X_{1}(z)=\frac{1}{2} \operatorname{Re}\left\{2 E(z)-\left(\beta^{2}+1\right) z+\frac{\beta^{2} c n z}{\operatorname{snz} d n z}\right\} ; \\
& X_{2}(z)=\frac{1}{2} \operatorname{Re} i\left\{2 E(z)+\left(\beta^{2}-1\right) z-\frac{\beta^{2} c n z}{\operatorname{snz} d n z}-4 E+2 K\right\} \\
& X_{3}(z)=\beta \log \left|\frac{\operatorname{sn} z}{\sqrt{2} d n z}\right|,
\end{aligned}
$$

where

$$
E(z)=\int_{0}^{z} d n^{2} \zeta d \zeta, \quad E=E(K), \quad \beta^{2}=8 \pi^{2} / \Gamma^{4}\left(\frac{1}{4}\right) .
$$

In particular $X_{3}(z)$ tends to $-\infty$ when $z \rightarrow 0$, to $+\infty$ when $z \rightarrow K+i K^{\prime}$ and to 0 when $z \rightarrow i K^{\prime}$ if we choose $\beta>0$.

Proof. The zeros and poles of $f$ and $g$ in a fundamental region may be depicted as

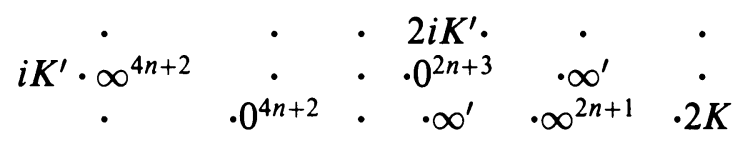

The only poles of $g$ in the fundamental region occur at $0, K$ and $K+i K^{\prime}$. The poles at 0 and $K+i K^{\prime}$ correspond to $p_{0}, p_{2}$ and $D=T^{2}-\left\{p_{0}, p_{2}, p_{3}\right\}$. The pole at $K$ is of order $2 n+1$ and $f$ has a zero at $K$ of order $4 n+2$ and no other zero in the fundamental region.

Consider next the residues of $f, f g$ and $f g^{2}$ at $0, K+i K^{\prime}$ and $i K^{\prime}$. We have $f(z),(f g)(z)$ and $\left(f g^{2}\right)(z)$ are even functions of $z$ and, as in the proof of Theorem 1, $f\left(z+i K^{\prime}\right),(f g)\left(z+i K^{\prime}\right)$ and $\left(f g^{2}\right)\left(z+i K^{\prime}\right)$ are also even functions of $z$. We also have $[8$, p. 503]

$$
\begin{aligned}
& s n\left(u+K+i K^{\prime}\right)=\frac{d n u}{k c n u}, \\
& c n\left(u+K+i K^{\prime}\right)=\frac{-i k^{\prime}}{k c n u}, \\
& d n\left(u+K+i K^{\prime}\right)=\frac{i k^{\prime} s n u}{c n u}
\end{aligned}
$$

which again imply that $f, f g$ and $f g^{2}$, as functions of $z+K+i K^{\prime}$, are even in $z$. Hence

$$
\operatorname{Res} f=\operatorname{Res} f g=\operatorname{Res} f g^{2}=0 \text { at } 0, K+i K^{\prime}, i K^{\prime} .
$$


Consider next the periods of $f, f g$ and $f g^{2}$. Let $\gamma_{1}$ and $\gamma_{2}$ be the two paths defined by (3.8) in the proof of Theorem 1 . We already have

$$
\int_{\gamma_{1}} f(z) d z=\int_{0}^{2 K} c n^{4 n+2} t d t \text { and } \int_{\gamma_{2}} f(z) d z=-i \int_{0}^{2 K} c n^{4 n+2} t d t .
$$

For the integral of $f g$ we need a bit of elementary trigonometry: The identities in (3.15) give for $n \geq 1$,

$$
\begin{aligned}
\frac{c n^{2 n+1} z}{\operatorname{snzdnz}=} & c n^{2 n+1} z\left(\frac{d n z}{s n z}+\frac{k^{2} s n z}{d n z}\right) \\
= & \frac{c n z d n z}{s n z}\left(1-s n^{2} z\right)^{n}+\frac{k^{2} c n z s n z}{d n z}\left(\frac{d n^{2} z-k^{\prime 2}}{k^{2}}\right)^{n} \\
= & \frac{c n z d n z}{s n z}+\sum_{\nu=1}^{n}\left(\begin{array}{l}
n \\
\nu
\end{array}\right)(-1)^{\nu} s^{2 \nu-1} z c n z d n z \\
& +k^{2}\left(\frac{-k^{\prime 2}}{k^{2}}\right)^{n} \frac{c n z s n z}{d n z} \\
& +\frac{1}{k^{2 n-2}} \sum_{\nu=1}^{n}\left(\begin{array}{l}
n \\
\nu
\end{array}\right)\left(-k^{\prime 2}\right)^{n-\nu} d n^{2 \nu-1} z \text { cn } z \text { sn } z .
\end{aligned}
$$

Since $(s n z)^{\prime}=c n z d n z$ and $(d n z)^{\prime}=-k^{2} \sin z c n z$, it follows that the integral of $f g$ contains two logarithmic terms plus two polynonials in $s n^{2} z$ and $d n^{2} z$ respectively. In view of the periodicity of $s n^{2} z$ and $d n^{2} z$ it follows that the value of $\operatorname{Re} \int f(z) g(z) d z$ on $\gamma_{j}$ depends on $\log |s n z|+$ $(-1)^{n+1} \frac{k^{\prime 2 n}}{k^{2 n}} \log |d n z|$. But again, by the "periodicity" of $s n z$ and $d n z$ namely, $s n(z+2 K)=-s n z$ and $d n(z+2 K)=d n z$, one sees that

$$
\operatorname{Re} \int_{\gamma_{j}} f(z) g(z) d t=0, \quad j=1,2 .
$$

For the integral of $f g^{2}$ we have

$$
\begin{aligned}
\int f(z) g^{2}(z) d z & =\beta^{2} \int \frac{1}{s n^{2} z d n^{2} z} d z \\
& =\beta^{2} \int\left(\frac{1}{s n^{2} z}+\frac{k^{2}}{d n^{2} z}\right) d z=\beta^{2}\left(z-\frac{c n z}{\operatorname{snz} z n z}\right)
\end{aligned}
$$

as may be verified by direct differentiation taking into account that $k^{2}=\frac{1}{2}$. Clearly then

$$
\int_{\gamma_{1}} f(z) g^{2}(z) d z=2 K \beta^{2} \text { and } \int_{\gamma_{2}} f(z) g^{2}(z) d z=2 i K^{\prime} \beta^{2} .
$$

In view of the definition of $\beta^{2}$ and the equality $K=K^{\prime}$ we have

$$
\overline{\int_{\gamma_{1}} f(z) d z}=\int_{0}^{2 K} c n^{4 n+2} t d t=2 K \beta^{2}=\int_{\gamma_{1}} f(z) g^{2}(z) d z
$$

and

$$
\overline{\int_{\gamma_{2}} f(z) d z}=i \int_{0}^{2 K} c n^{4 n+2} t d t=2 i K \beta^{2}=\int_{\gamma_{2}} f(z) g^{2}(z) d z .
$$


Completeness follows immediately as in Theorem 1 and so $X$ defined by the Weierstrass representation with $f$ and $g$ is a regular conformal minimal immersion and $M=X(D)$ is complete. Finally, since $g$ is of order $2 n+3$, the curvature of $M$ will be $-4 \pi(2 n+3)$.

(b) If $n=0$, then "starting" all integrals at $K$ we have

$$
\begin{aligned}
& \int_{K}^{z} f(\zeta) d \zeta=\int_{K}^{z} c n^{2} \zeta d \zeta=2 E(z)-z-2 E+K \\
& \operatorname{Re} \int_{K}^{z} f(\zeta) g(\zeta) d \zeta=\operatorname{Re} \beta \int_{K}^{z}(c n \zeta / \operatorname{sn} \zeta d n \zeta) d \zeta \\
& =\beta \log |\operatorname{sn} z / d n z|-\beta \log \sqrt{2} \\
& \int_{K}^{z} f(\zeta) g^{2}(\zeta) d \zeta=\beta^{2}\left(z-\frac{c n z}{\operatorname{sn} z d n z}-K\right)
\end{aligned}
$$

It follows that

$$
\begin{aligned}
X_{1}(z) & =\frac{1}{2} \operatorname{Re} \int_{K}^{z} f\left(1-g^{2}\right) d \zeta \\
& =\frac{1}{2} \operatorname{Re}\left\{2 E(z)-\left(\beta^{2}+1\right) z+\beta^{2} \frac{c n z}{\operatorname{snz} d n z}-2 E+\left(\beta^{2}+1\right) K\right\}, \\
X_{2}(z) & =\frac{1}{2} \operatorname{Re} i \int_{K}^{z} f\left(1+g^{2}\right) d \zeta \\
& =\frac{1}{2} \operatorname{Re} i\left\{2 E(z)+\left(\beta^{2}-1\right) z-\beta^{2} \frac{c n z}{\operatorname{sn} z d n z}-2 E-\left(\beta^{2}-1\right) K\right\}, \\
X_{3}(z) & =\operatorname{Re} \int_{K}^{z} f(\zeta) g(\zeta) d \zeta=\beta \log \left|\frac{\operatorname{sn} z}{\sqrt{2} d n z}\right| .
\end{aligned}
$$

On the other hand, the value of the constant $\beta$ as defind in (4.2) is

$$
\beta^{2}=\frac{1}{2 K} \int_{0}^{2 K} c n^{2} t d t=\frac{1}{2 K}\{2 E(2 K)-2 K\}=\frac{2 E-K}{K},
$$

which implies that $\beta^{2}+1=2 E / K$ or $K\left(\beta^{2}+1\right)=2 E$ thus removing the constant term in the expression for $X_{1}$ and simplifying the constant term $-2 E-\left(\beta^{2}-1\right) K$ in $X_{2}$ to $2 K-4 E$. The explicit evaluation of $\beta^{2}$ is found in $[8$, p. 525]. Finally as $z \rightarrow 0, s n z \rightarrow 0$ and $d n z \rightarrow 1 / \sqrt{2}$ so that, since $\beta>0, X_{3} \rightarrow-\infty$. Similarly $X_{3} \rightarrow+\infty$ when $z \rightarrow K+i K^{\prime}$. When $z \rightarrow i K^{\prime}$ the ratio $\frac{s n z}{d n z}$ approaches $i \sqrt{2}$; hence $X_{3} \rightarrow 0$ in this case. Let us note that for each $n$ the components $X_{1}, X_{2}$ and $X_{3}$ of the corresponding surface may be computed in terms of $E(z), s n z, c n z, d n z$ and other simple functions. Indeed, all that is needed is a recurrence relation for the integral of $c n^{4 n+2}$, but this is easily obtained by two differentiations. The same, of course, is true of integrals involving powers of $s n$ and $d n$.

\section{SYMMETRY}

In order to study the symmetries of the surfaces obtained we shall take as fundamental region the square $F$ with vertices $-i K^{\prime}, 2 K-i K^{\prime}, 2 K+i K^{\prime}, i K^{\prime}$ where we continue to write $K^{\prime}$ although now $K^{\prime}=K$ since $k=1 / \sqrt{2}=k^{\prime}$. Following Hoffman and Meeks [4] we introduce the following transformations 
of the above square:

$\beta(K+z)=K+\bar{z}$ reflection about $x$-axis;

$\rho^{m}(K+z)=K+i^{m} z$ rotation by $m \pi / 2$ about $K, m=1,2,3$;

$\alpha(K+z)=\rho(\beta(K+z))=K+i \bar{z}$ reflection through positive

diagonal through $K$;

$\hat{\alpha}(K+z)=\rho^{2}(\beta(K+z))=K-\bar{z}$ reflection through vertical line through $K$;

$\mu(K+z)=\rho^{3}(\beta(K+z))=K+i \bar{z}$ reflection through negative diagonal

$$
\begin{array}{cccc}
i K^{\prime} & \cdot & \cdot & \bullet \\
0 & \cdot & \cdot K & \bullet
\end{array}
$$

Note that, although we are using the same notation, the transformations $\alpha, \rho, \beta, \mu$ above are not, strictly speaking, the same as those in [4]. The next lemma translates well-known periodicity properties of the Jacobian elliptic functions into geometric language.

Lemma 1. Let $s n z, c n z$ and $d n z$ be the Jacobian elliptic functions to the modulus $k=\frac{1}{\sqrt{2}}$. Then

$$
\begin{array}{ll}
c n(\mu(K+z))=i \overline{c n(K+z)}, & c n(\rho(K+z))=i c n(K+z), \\
c n(\beta(K+z))=\overline{c n(K+z)}, & c n(\hat{\alpha}(K+z))=\overline{-c n}(K+z)
\end{array}
$$

and

$$
\begin{aligned}
& \text { sndn } \mu(K+z)=\overline{\operatorname{sndn}}(K+z), \quad \text { sndn } \rho(K+z)=\operatorname{sndn}(K+z) \text {, } \\
& \operatorname{sndn} \beta(K+z)=\overline{\operatorname{sndn}}(K+z), \quad \operatorname{sndn\hat {\alpha }}(K+z)=\overline{\operatorname{sndn}}(K+z) \text {. }
\end{aligned}
$$

Proof. All three Jacobian functions are real on the real axis and

$$
\operatorname{sn}(i z)=i \operatorname{sn} z / c n z, \quad c n(i z)=1 / c n z, \quad d n(i z)=d n z / c n z
$$

by the Jacobian imaginary transformation with $k=k^{\prime}$. If we recall (3.20), it becomes an easy matter to verify the following:

$$
\begin{aligned}
c n(\mu(K+z)) & =c n(K+i \bar{z})=-k^{\prime} \operatorname{sn}(i \bar{z}) / d n(i \bar{z})=-k^{\prime} i \operatorname{sn}(\bar{z}) / d n(\bar{z}) \\
& =i \overline{\left(-k^{\prime} \operatorname{sn} z / d n z\right)}=i \overline{c n(K+z)} .
\end{aligned}
$$

The other relations in (5.1) are obtained in a similar fashion. The computations leading to (5.2) are completely analogous.

The next step is to use Lemma 1 to obtain geometric statements about the functions $f$ and $g$ of Theorems 1 and 2. Since, in part (b), of Theorem 1 the constant $\alpha$ is pure imaginary we expect a difference in this case.

Lemma 2. Let $f$ and $g$ be the pair of functions defined in Theorem 1 or Theorem 2. Then

$$
\begin{gathered}
f(\mu(K+z))=-\bar{f}(K+z), \quad f(\rho(K+z))=-f(K+z), \\
f(\beta(K+z))=\bar{f}(K+z) \\
f g^{2}(\mu(K+z))=-\overline{f g^{2}}(K+z), \quad f g^{2}(\rho(K+z))=-f g^{2}(K+z), \\
f g^{2}(\beta(K+z))=\overline{f g^{2}}(K+z)
\end{gathered}
$$




$$
f g(\rho(K+z))=(-1)^{n} i f g(K+z), \quad f g(\beta(K+z))=\varepsilon \overline{f g}(K+z)
$$

where $\varepsilon=+1$ if $\alpha$ is real and $\varepsilon=-1$ if $\alpha$ is pure imaginary.

Proof. All relations follow immediately from Lemma 1 and the definitions of $f$ and $g$. For example, if $f$ and $g$ are defined by (3.2), then

$$
f g(\beta(K+z))=\alpha\left(\overline{c n}^{2 n+1} \overline{s n} \overline{d n}\right)(K+z)=(\alpha / \bar{\alpha}) \overline{f g}(K+z)=\varepsilon \overline{f g}(K+z) .
$$

Let us now introduce, again following Hoffman and Meeks [4], two orthogonal motions of $\mathbb{R}^{3}$ defined by

$$
B_{\varepsilon}=\left[\begin{array}{ccc}
1 & 0 & 0 \\
0 & -1 & 0 \\
0 & 0 & \varepsilon
\end{array}\right], \quad R_{n}=\left[\begin{array}{ccc}
0 & -1 & 0 \\
1 & 0 & 0 \\
0 & 0 & (-1)^{n+1}
\end{array}\right]
$$

where $\varepsilon= \pm 1$ and $n$ is an integer. If $\varepsilon=+1$, then $B_{\varepsilon}$ is reflection in the $\left(x_{1}, x_{3}\right)$-plane. If $\varepsilon=-1, B_{\varepsilon}$ is a product of two reflections or a rotation by $\pi$ about the $x_{1}$-axis. If $n$ is odd, $R_{n}$ is a rotation by $\pi / 2$ about the $x_{3}$-axis; if $n$ is even, it is a rotation by $\pi / 2$ about the $x_{3}$-axis followed by a reflection in the $\left(x_{1}, x_{2}\right)$-plane. It is easy to verify that $B_{\varepsilon}^{2}=R_{n}^{4}=I$ and, in fact, that $B_{\varepsilon}$ is of order 2 and $R_{n}$ is of order 4 as elements of the group of orthogonal motions of $\mathbb{R}^{3}$. Let $G_{\varepsilon, n}$ be the group generated by $B_{\varepsilon}$ and $R_{n}$. Then $G_{\varepsilon, n}$ is the dihedral group with eight elements. We may consider $G_{\varepsilon, n}$ as acting on $\mathbb{R}^{3}$ through the motions $B_{\varepsilon}$ and $R_{n}$. We may also identify $B_{\varepsilon}$ and $R_{n}$ with the transformations $\beta$ and $\rho$ of the square $F$. Then $G_{\varepsilon, n}$ may be considered as acting on the square $F$.

Recall that $F$ is the square with vertices $-i K^{\prime}, 2 K-i K^{\prime}, 2 K+i K^{\prime}$ and $i K^{\prime}$ and $p_{0}, p_{1}, p_{2}$ and $p_{3}$ are the points on the torus $T^{2}$ corresponding to the points $0, K, K-i K^{\prime}$ and $-i K^{\prime}$ respectively. $D$ is the domain occurring in Theorem 1 or Theorem 2 i.e. $D=T^{2}-\left\{p_{3}\right\}$ or $D=T^{2}-\left\{p_{1}, p_{3}\right\}$ or $D=T^{2}-\left\{p_{0}, p_{2}, p_{3}\right\} . X: D \rightarrow \mathbb{R}^{3}$ is the immersion defined by the Weierstrass representation with the functions $f$ and $g$. Since $f$ and $g$ involve an integer $n$ and a constant $\alpha=\alpha(n)$, so does $X$. In other words $X=X_{n, \alpha}$ or $X_{n, \varepsilon}$ where $\varepsilon=+1$ if $\alpha$ is real and $\varepsilon=-1$ if $\alpha$ is pure imaginary. In the next result, we refer to $n$ and $\varepsilon$ as the indices corresponding to the surface $X$ described in Theorems 1 and 2. The next result is identical to the result of Hoffman and Meeks [4, p. 117].

Theorem 3. Let $M=X(D)$ be the minimal surface described in Theorem 1 or Theorem 2, and let $n$ and $\varepsilon$ be the corresponding indices. Let $G$ be the dihedral group, with eight elements. Then $G$, acting on $\mathbb{R}^{3}$, is a symmetry group of $M=X(D) \subset R^{3}$. The immersion $X: D \rightarrow \mathbb{R}^{3}$ is compatible with the action of $G$ on $D$. That is,

$$
X \circ \rho=R_{n} \circ X \text { and } X \circ \beta=B_{\varepsilon} \circ X \text {. }
$$

In the metric on $D$ induced by $X, G$ acts by isometries . 
Proof. We first translate the properties of $f, f g, f g^{2}$ in Lemma 2 into properties of $\phi_{1}=f\left(1-g^{2}\right) / 2, \phi_{2}=i f\left(1+g^{2}\right) / 2$ and $\phi_{3}=f g$. We have

$$
\begin{aligned}
\phi_{1}(\rho(K+z)) & =\frac{1}{2} f\left(1-g^{2}\right)(\rho(K+z)) \\
& =\frac{1}{2}\left(-f-f g^{2}\right)(K+z)=i \phi_{2}(K+z) ; \\
\phi_{2}(\rho(K+z)) & =\frac{i}{2} f\left(1+g^{2}\right)(\rho(K+z))=-i \phi_{1}(K+z) ; \\
\phi_{3}(\rho(K+z)) & =(-1)^{n} i \phi_{3}(K+z) .
\end{aligned}
$$

Thus

$$
\begin{aligned}
\left(X_{1}, X_{2}\right)(\rho(K+z))=\left(X_{1}, X_{2}\right)(K+i z)=\frac{1}{2} \operatorname{Re} \int_{K}^{K+i z}\left(\phi_{1}, \phi_{2}\right)(\zeta) d \zeta \\
=\frac{1}{2} \operatorname{Re} \int_{0}^{z}\left(\phi_{1}, \phi_{2}\right)(K+i \zeta) i d \zeta=\frac{1}{2} \operatorname{Re} \int_{0}^{z}\left(-\phi_{2}, \phi_{1}\right)(K+\zeta) d \zeta \\
=\frac{1}{2} \operatorname{Re} \int_{K}^{K+z}\left(-\phi_{2}, \phi_{1}\right)(\zeta) d \zeta=\left(-X_{2}, X_{1}\right)(K+z) .
\end{aligned}
$$

Also

$$
\begin{aligned}
X_{3}(\rho(K+z)) & =X_{3}(K+i z)=\operatorname{Re} \int_{K}^{K+i z} \phi_{3}(\zeta) d \zeta=\operatorname{Re} \int_{0}^{z} \phi_{3}(K+i \zeta) i d \zeta \\
& =\operatorname{Re} \int_{0}^{z}(-1)^{n+1} \phi_{3}(K+\zeta) d \zeta=(-1)^{n+1} X_{3}(K+z) .
\end{aligned}
$$

It follows that

$$
X \circ \rho=R_{n} \circ X
$$

where

$$
R_{n}=\left[\begin{array}{ccc}
0 & -1 & 0 \\
1 & 0 & 0 \\
0 & 0 & (-1)^{n+1}
\end{array}\right]
$$

Again,

$$
\begin{aligned}
& \phi_{1}(\beta(K+z))=\frac{1}{2} f\left(1-g^{2}\right)(\beta(K+z))=\frac{1}{2}\left(\bar{f}-\overline{f g^{2}}\right)(K+z)=\bar{\phi}_{1}(K+z), \\
& \phi_{2}(\beta(K+z))=\frac{i}{2} f\left(1+g^{2}\right)(\beta(K+z))=\frac{i}{2}\left(\bar{f}-\overline{f g}^{2}\right)(K+z)=-\phi_{2}(K+z), \\
& \phi_{3}(\beta(K+z))=f g(\beta(K+z))=\varepsilon \overline{f g}(K+z)=\varepsilon \phi_{3}(K+z)
\end{aligned}
$$

where $\varepsilon=+1$ if $\alpha$ is real and $\varepsilon=-1$ if $\alpha$ is pure imaginary.

It follows that

$$
\begin{aligned}
\left(X_{1},\right. & \left.X_{2}\right)(\beta(K+z))=\left(X_{1}, X_{2}\right)(K+\bar{z})=\frac{1}{2} \operatorname{Re} \int_{K}^{K+\bar{z}}\left(\phi_{1}, \phi_{2}\right)(\zeta) d \zeta \\
= & \frac{1}{2} \operatorname{Re} \int_{0}^{z}\left(\phi_{1}, \phi_{2}\right)(K+\bar{\zeta}) d \bar{\zeta}=\frac{1}{2} \operatorname{Re} \int_{0}^{z}\left(\bar{\phi}_{1}, \bar{\phi}_{2}\right)(K+\zeta) d \bar{\zeta} \\
= & \frac{1}{2} \operatorname{Re} \int_{K}^{K+z}\left(\bar{\phi}_{1},-\bar{\phi}_{2}\right)(\zeta) d \bar{\zeta}=\frac{1}{2} \operatorname{Re} \int_{K}^{K+z}\left(\phi_{1},-\phi_{2}\right)(\zeta) d \zeta \\
= & \left(X_{1},-X_{2}\right)(K+z) .
\end{aligned}
$$




$$
\begin{aligned}
X_{3}(\beta(K+z)) & =\operatorname{Re} \int_{K}^{K+\bar{z}} \phi_{3}(\zeta) d \zeta=\operatorname{Re} \int_{0}^{z} \phi_{3}(K+\bar{\zeta}) d \bar{\zeta} \\
& =\operatorname{Re} \int_{0}^{z} \varepsilon \bar{\phi}_{3}(K+\zeta) d \bar{\zeta}=\varepsilon \operatorname{Re} \overline{\int_{0}^{z} \phi_{3}}(K+\zeta) d \zeta=\varepsilon X_{3}(K+z)
\end{aligned}
$$

Thus we have

$$
X \circ \beta=B_{\varepsilon} \circ X
$$

where

$$
B_{\varepsilon}=\left[\begin{array}{ccc}
1 & 0 & 0 \\
0 & -1 & 0 \\
0 & 0 & \varepsilon
\end{array}\right]
$$

This is all that is necessary to establish and the proof of Theorem 3 is completed.

It is, of course, possible to go into more detail and study the mapping properties of the immersion. Since this is more or less straightforward, we omit such considerations. Let us, however, point out one simple instance, say, when $\alpha$ is real: since $c n$ and $d n$ are real on the real and imaginary axes while $s n$ is real on the real axis and pure imaginary on the imaginary axis, it follows that $f, f g^{2}$ are real on both the real and imaginary axes in case $\alpha$ is real. Thus the segments $0, \overrightarrow{2} K$ and $-i K^{\prime}, i K^{\prime}$ are mapped into the $\left(x_{1}, x_{3}\right)$-plane. Similar statements can be made in case $\alpha$ is imaginary and for other segments in the square $F$. Let us note finally that as an immediate corollary of Theorem 3 we have that if $n$ is even and $\varepsilon=1$, the surface $M$ is made up of eight congruent pieces. Again similar statements can be made in the other cases.

\section{REFERENCES}

1. L. Barbosa and G. Colares, Minimal surfaces in $\mathbb{R}^{3}$, Lecture Notes in Math., vol. 1195, Springer-Verlag, Berlin and New York, 1986.

2. C. Costa, Example of a complete minimal immersion in $\mathbb{R}^{3}$ of genus one and three embedded ends, Bol. Soc. Brasil Mat. 15 (1984), 47-54.

3. C. C. Chen and F. Gackstätter, Elliptische und hyperelliptische Funktionen und vollständige Minimalflächen vom Enneperschen Typ., Math. Ann. 259 (1982), 359-369.

4. D. Hoffman and W. Meeks III, A complete embedded minimal surface in $\mathbb{R}^{3}$ with genus one and three ends, J. Differential Geom. 21 (1985), 109-127.

5. L. Jorge and W. Meeks III, The topology of complete minimal surfaces of finite total Gaussian curvature, Topology 22 (1983), 203-221.

6. R. Osserman, A survey of minimal surfaces, 2nd ed., Dover, New York, 1986.

7. M. Wohlgemuth, Abelsche Minimalflaschen, Diplomarbeit Universität Bonn, 1987.

8. E. T. Whittaker and G. N. Watson, A course of modern analysis, 4th ed., Cambridge Univ. Press, London and New York, 1927.

Department of Mathematical Sciences, Purdue University, West Lafayette, Indiana 47907

Current address: Department of Mathematics, American University of Beirut, 850 Third Ave., New York, New York 10022 